\title{
MATH SIMULATION OF CONTACT INTERACTION DURING SPACECRAFT DOCKING AND ROBOTIC ASSEMBLY OPERATIONS
}

\author{
A. Yaskevich \\ Rocket Space Corporation "Energia" \\ Korolev city, Russia \\ Andrey.Yaskevich@rsce.ru
}

Keywords: Docking and berthing interfaces, Contact interaction, Real-time math simulation.

Abstract. Contact interaction math models can be split into two classes depending on the character of the relative motion of assembly interface contacting elements.

In the first-class models, one of contacting elements has several degrees of relative motion freedom. Therefore contact reaction forces must be taken into account in relative motion dynamics equations of a mechanical system. In this case the most efficient method of calculating such forces is based on the assumption of a contact penetration counteracted by contact stiffness. The penetration value is controlled; its maximal value determines the geometric contact simulation error. For the first-class contact models, basic principles of development and a classification of main pairs of interacting elements are presented; algorithms for calculation of penetration values, reaction unit vectors at contact points and contact forces are described.

In the second-class models, one of contacting elements has only one degree of freedom, and its relative displacement is counteracted by a spring. Here, a slight inertia of such an element does not influence the motion dynamics of mating interfaces, but it is only the spring force that is taken into account. Therefore contact reaction forces in the second-class models are calculated based on spring deformation only, without using contact penetration. In this case the geometric place of contact, which determines the spring deformation, is calculated by the iterative method with any given accuracy. This feature is particularly useful for simulating contact mechanisms that provide a stiff interface joint and have very small displacements. Models of this class are also used for describing the motion of capture latches.

Surfaces of the second and forth order (cone, torus) are approximated by more simple geometric elements of the first and third order respectively (segment, sphere) in contact models of both classes. As a result, a multiple use of simple analytical relations replaces a solution of a complex geometric problem. Calculation efficiency is ensured by the dichotomy method, as well as by a preliminary generation of the highest possible number of parameters that are just transformed during simulation into the coordinate system of the geometric problem solution.

The described algorithms provide a real-time simulation of contact interaction of different mating interfaces i.e. probe-cone type and androgynous peripheral docking units, specialized berthing devices. 


\section{INTODUCTION}

Spacecraft docking or berthing represents an in-orbit mechanical assembly process that starts at the moment of their first contact and ends with a formation of a stiff joint. It is implemented with the use of active and passive docking assemblies that have mechanisms and interfaces with contacting surfaces. Forces and moments created by the spacecraft control system, mechanisms and contact reactions ensure a decrease of relative linear and angular misalignments at different phases of docking/berthing. A complex form of contacting surfaces can be represented by a group of elements whose geometry is described by equations of the first (line, plane) to forth (torus) order. Contact interaction math models can be split into two classes depending on the character of the relative motion of mating interface contact elements.

In the first-class models, it is considered that an interface element, e.g. a docking mechanism buffer link, has several degrees of freedom of a relative motion described by differential equations that involve contact reaction forces. To calculate these forces, the most efficient method is used, which is based on the admission of a contact penetration counteracted by contact stiffness. The penetration value is controlled; its maximal value determines the geometric contact simulation error.

In the second-class models, one of contacting elements has only one degree of freedom, and its relative displacement is counteracted by a spring. Here, a slight inertia of such an element does not influence the motion dynamics of mating interfaces, but it is only the spring tension considered that forces this element to take either its initial position (the spring is not deformed) or a position corresponding to a contact with the guide surface (the spring is deformed). This class includes the pairs latch/sensor - guide surface and active - passive hook. Contact parameters for this class pairs can be obtained in a basically different way, i.e. by iterative methods based on only geometric relations with any given accuracy that does not depend on the integration step value in the motion dynamic equations of mating spacecraft and docking mechanisms. This feature is particularly useful for simulating contact of mechanisms that provide a stiff interface joint and have very small displacements.

This research brings forward efficient calculation algorithms of analysis of contact conditions and determination of contact parameters for both classes of contacting elements based on simple analytical relations. They allow development of detailed and adequate models that can be computed real-time.

\section{CONTACT ELEMENTS GEOMETRY DESCRIPTION}

Development of a mathematical description of contact interaction of guide surface elements of docking assemblies is based on the following main points.

1. In a mathematical simulation each contact area is replaced by a single point. The number of contact points can be arbitrary, but must be finite.

2. Types of pairs of guide surface contacting elements make a finite set. Each of them is represented by a finite aggregation of geometric primitives, for which contact conditions and geometric parameters are determined by analytical expressions. As a result, a multiple use of simple analytical relations replaces a solution of a complex geometric problem.

3. Each analytical solution is developed subject to fulfillment of the designed allowable range of lateral and angular misalignments of docking assemblies. A violation of this range means an off-nominal course of the operation and leads to a termination of the simulation.

Structural analysis of different types of mating interfaces (see Fig. 1) allows sorting out the following pairs of contacting elements of guide surfaces:

- spherical surface of the head and contact sensors of the probe - receiving cone surface;

- spherical surface of the probe head and contact sensors - receiving socket cylinder border; 

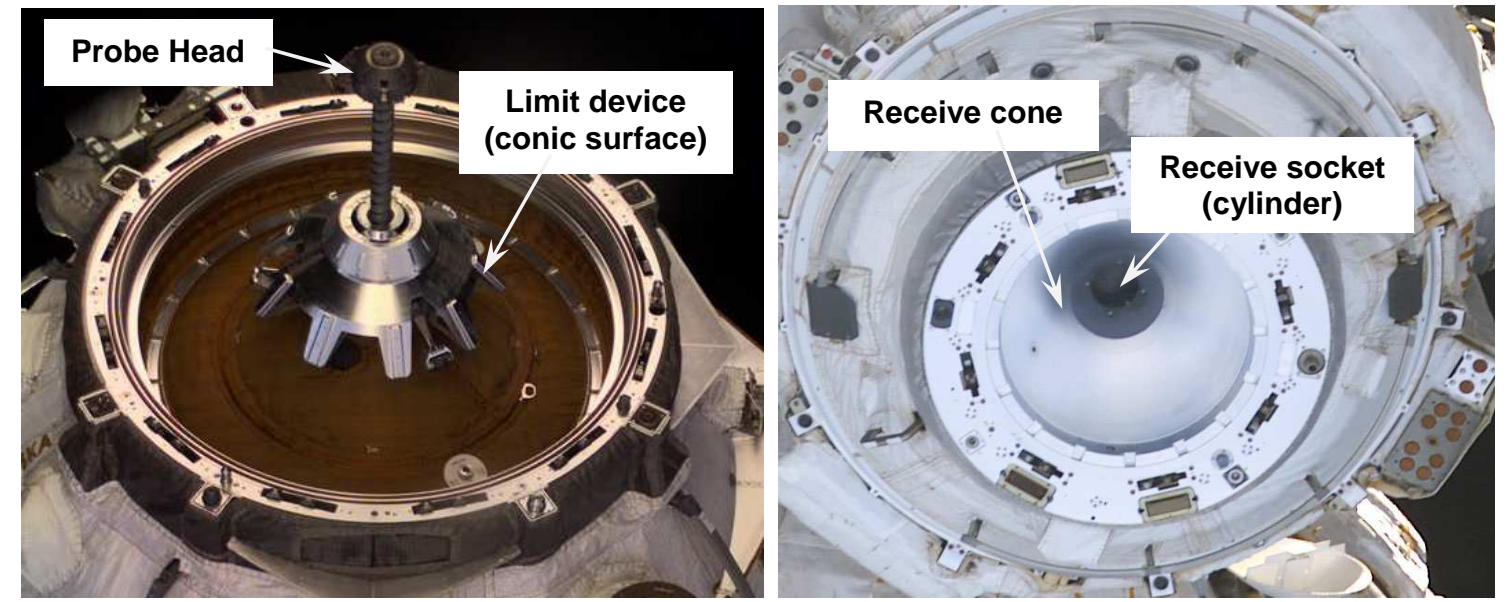

a
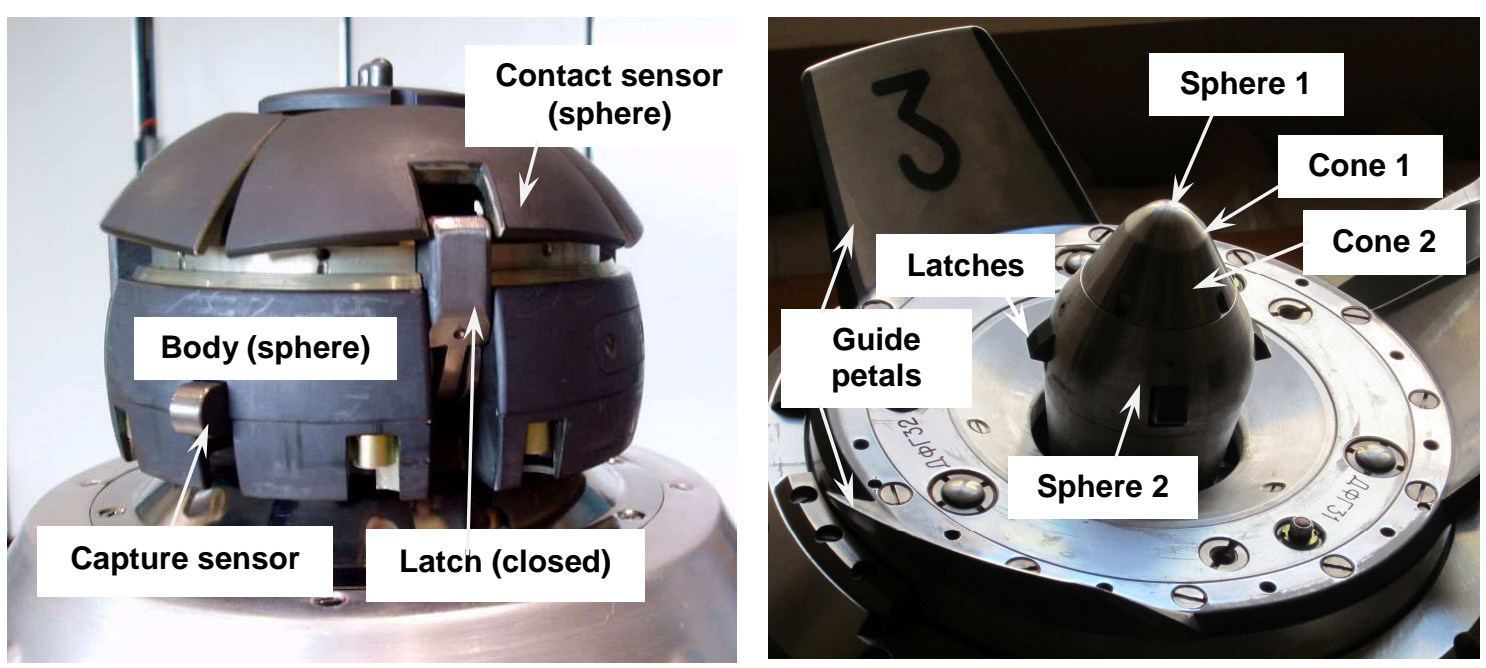

b

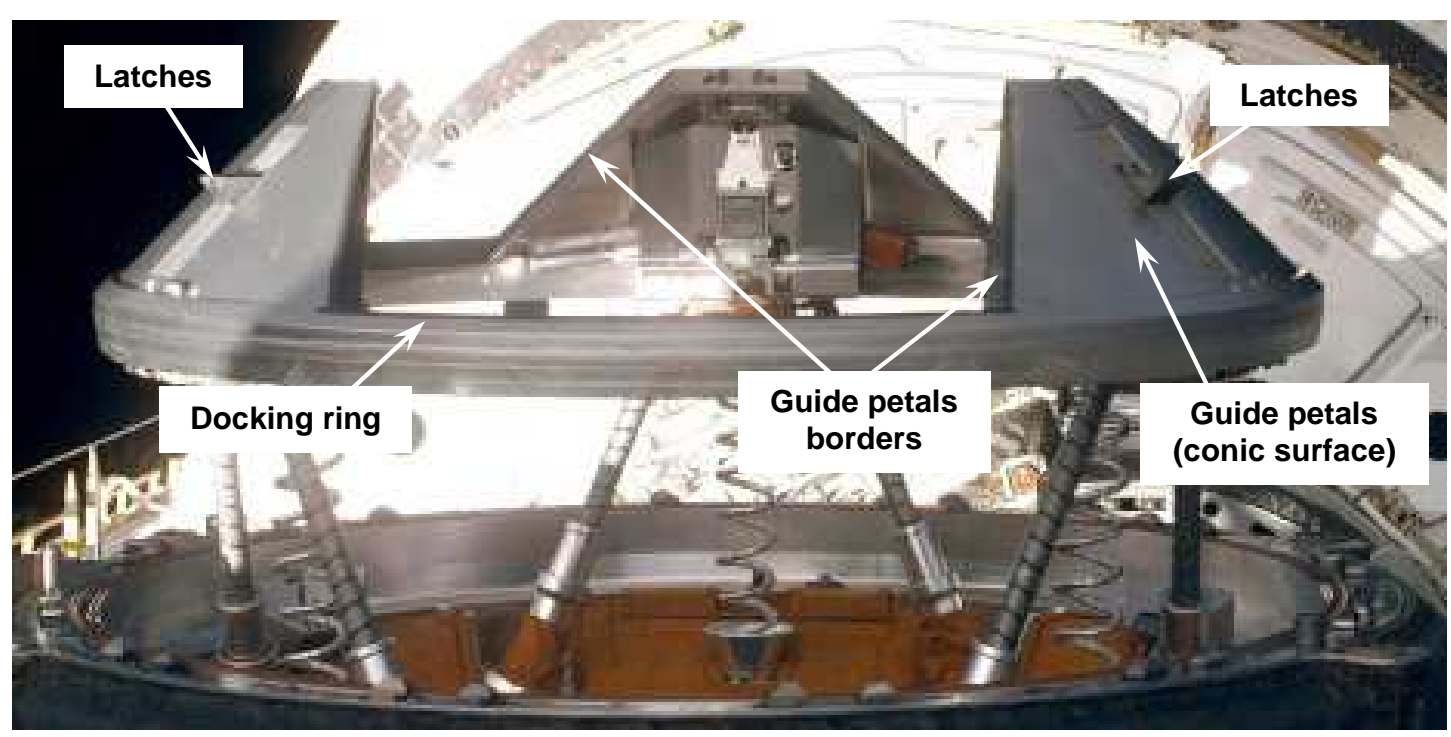

C

Fig. 1. Types of contact surfaces of docking interfaces -Probe-Cone docking units (a); Probe head and capture head of berthing unit (b), androgynous docking unit (c) 
- spherical surface of the probe head and contact sensors - receiving socket cylinder surface;

- spherical surface of the probe head - receiving socket bottom;

- capture head conic surface base - receiving cone surface;

- capture head conic surface - receiving socket cylinder border (circle);

- capture head conic surface base - receiving socket cylinder surface;

- docking mechanism limit device - receiving cone base border;

- docking mechanism limit device extremity- receiving cone surface;

- aligning lever roller- receiving cone surface;

- latch extremity- receiving cone surface;

- latch and capture sensor on the probe head - receiving socket cylinder border;

- guide petal conic surface fragment - receiving cone base border;

- guide petals lateral borders of active and passive assemblies;

- guide petals border - receiving cone base border;

- torus surface of the lock head- conic receiving cone surface;

- torus surface of the lock head - receiving socket cylinder border (circle);

- torus surface of the lock head - receiving socket cylinder surface.

Guide petals are located at the periphery of docking assemblies, they are intended for decreasing lateral and angular misalignments during their axial approach. Geometrically they represent trapezoidal fragments of round conic surfaces with the angle between their axis and generatrix equal to $45^{\circ}$. The inclined lateral borders of guide petals ensue from the sectioning of the conic surface by planes, i.e. lines of the second order, in particular hyperbolas. Despite this, they are represented by line segments; the accuracy of the math model is determined by their maximal distance from the surface. If a lateral border of the guide petal is a stiffening rib it can be specified by two segments as its contact borders. The dimensions and relative position of the guide petals allowed by initial approach conditions make their contact possible with their borders only, and with only one border for each petal.

The above mentioned pairs of guide surface contacting elements can be described by the following main pairs of geometric primitives, for which contact conditions and geometric parameters are determined by analytical expressions:

- $\quad$ sphere - truncated receiving cone;

- $\quad$ sphere - circle;

- $\quad$ sphere - cylinder;

- $\quad$ sphere - plane;

- $\quad$ point (extremity of a line segment) - truncated receiving cone;

- $\quad$ point (extremity of a line segment) - cylinder;

- $\quad$ line segment - line segment;

- $\quad$ line segment - plane;

- $\quad$ line segment - circle;

- $\quad$ inclined lateral borders of guiding petals.

More complex elements of contacting surfaces are represented by finite sets of geometric primitives. Thus, the surface of a truncated cone or its fragments contacting with a circle are replaced by a finite set of generatrix segments of a constant or variable length. Surface elements in the form of a torus are represented by a finite aggregation of spherical surfaces (spheres). The number of approximating geometric primitives is determined on the basis of the required accuracy. 


\section{FIRST CLASS MODELS FOR CONTACT FORCES CALCULATION}

\subsection{Taking into account unilateral constraints in first-class contact models}

Contact of two and more bodies that include guide surfaces is hereinafter considered not as a shock characterized by instant change of velocities [1] but as a dynamic process, in which unilateral constraints are imposed on the motion of the bodies. Physically, such constraints are realized through relevant reaction forces. In a mathematical simulation these constraints can be taken into account in two ways - with a constraint equation or through a direct calculation of contact interaction forces.

If equations of temporarily imposed unilateral constraints are used they are to be written relative to accelerations

$$
\mathbf{H} \ddot{\mathbf{q}}+\mathbf{h}(\mathbf{q}, \dot{\mathbf{q}})=\mathbf{0}
$$

and introduced into mechanical system dynamic equations

$$
\left[\begin{array}{cc}
\mathbf{A}(\mathbf{q}) & \mathbf{H}^{T} \\
\mathbf{H} & \mathbf{0}
\end{array}\right]\left[\begin{array}{c}
\ddot{\mathbf{q}} \\
\lambda
\end{array}\right]=\left[\begin{array}{c}
\mathbf{b}(\mathbf{q}, \dot{\mathbf{q}})+\mathbf{Q} \\
-\mathbf{h}(\mathbf{q}, \dot{\mathbf{q}})
\end{array}\right]
$$

where $\mathbf{H}-(m \times n)-$ matrix; $\mathbf{q}-(n \times 1)-$ vector of generalized coordinates; $\boldsymbol{\lambda}-(m \times 1)-$ vector of Lagrange multipliers; $\mathbf{A}(\mathbf{q})-(n \times n)-$ generalized inertia matrix; $\mathbf{b}(\mathbf{q}, \dot{\mathbf{q}})-(n \times 1)-$ vector of generalized inertia forces; $\mathbf{Q}-(n \times 1)-$ generalized forces vector.

This method of constraint allowance has low computational efficiency for the following reasons. First, the system of equations (2) can be solved relative to $\ddot{\mathbf{q}}$ and $\lambda$ only without linearly dependent lines in (1). In practice, the number of contacts can be great, and to fulfill this requirement decision algorithms for linearly dependent constraint equations are to be used. Second, the system of equations (2) is differential-algebraic. To solve it, methods [2-4], more complex than an explicit integration of dynamic equations without using (1) and (2), are to be applied.

Hereinafter we use a more computationally efficient method for motion constraint allowance based on a direct calculation of the contact reaction force using the simple relation $\mathbf{f}_{R}=\left(k_{C} \Delta_{n}+c_{C} \dot{\Delta}_{n}\right) \mathbf{n}_{R}$, in which the value of contact deformation $\Delta_{n}$ and its velocity $\dot{\Delta}_{n}$ are determined along the normal unit vector $\mathbf{n}_{R}$ which is common for the contacting surfaces; $k_{C}$ and $c_{C}-$ contact rigidity and damping factor. This method is mathematically equivalent to the regularization method [2] that uses a virtual oscillator but does not require a calculation of coefficients and exclusion of dependent constraint equations or an integration of differential-algebraic equations. Contact reaction forces enter into the right part of the mechanical system dynamic equations.

Penetration of an active geometric primitive into a passive one along their common normal vector at the contact point determines the value of the contact reaction force in the mathematical model. The value of the contract penetration is controlled, if its allowable limit is exceeded the simulation process is terminated with a corresponding diagnostic message.

Geometric parameters of the contact point are its coordinates, components of the contact reaction force unit vector normal to the contacting primitives, and the value of the penetration along this vector; kinematic parameters are the penetration velocity and the unit velocity vector of the active contacting primitive relative to the passive one in the contact plane passing through the contact point. Dynamic parameters are components of the force applied at 
the contact point, i.e. a normal component (penetration reaction) and a tangential one (friction force).

Expressions for contact conditions and parameters are built in the coordinate system $X_{P} Y_{P} Z_{P}$ of the passive assembly, where the $X_{P}$ axis corresponds to the direction of approach. Parameters of active geometric primitives are expressed in the coordinate systems of the active assembly mechanism links and converted into $X_{P} Y_{P} Z_{P}$, and parameters of passive ones are constant values calculated once.

Given below are some main algorithms of calculation of geometric contact parameters. In the simplest ones, the active geometric primitive is a sphere with the radius $R_{S}$, which center $P_{S}$ in $X_{P} Y_{P} Z_{P}$ is determined by the vector $\mathbf{r}_{S}^{(P)}=\left[x_{S}, y_{S}, z_{S}\right]^{T}$. This is specified by the symmetry of the sphere relative to its center and the axial symmetry of the counterpart geometric primitives, i.e. a truncated cone, a circle and a cylinder. Due to the equal probability of the directions of lateral and angular misalignments, $X_{P}$ is the symmetry axis of geometric primitives of passive guide elements, i.e. a receiving cone, a cylinder and a circle. As the direction of this axis corresponds to the active vehicle approach, therefore:

- the vector from the base to the top of the receiving cone coincides with the direction of this axis (misalignments should decrease at approach);

- the contact reaction vector that reflects the impact of the active guide elements on the passive ones has a positive projection on this axis.

All the above mentioned geometric characteristics are taken into account when forming analytical expressions that determine contact conditions and parameters. The direction of the contact reaction unit vector $\mathbf{n}_{R}=\left[n_{X}, n_{Y}, n_{Z}\right]^{T}$ coincides with the direction of the sphere radius, and the position of the contact point is determined by the vector $\mathbf{r}_{P, k}^{(P)}=\left[x_{S}+n_{X} R_{S}, y_{S}+n_{Y} R_{S}, z_{S}+n_{Z} R_{S}\right]^{T}$.

\subsection{Calculation of sphere - truncated receiving cone surface contact parameters.}

Parameters of a passive truncated cone are radii $R_{C B}$ and $R_{C T}$ of the lower and upper bases, the $\alpha_{C}$ angle between the axis and the generatrix, and the $P_{T}$ point of intersection of its generatrices, which is determined by the vector $\mathbf{r}_{T}^{(P)}=\left[x_{T}, 0,0\right]^{T}$ (see Fig. 2). To speed up calculations, a preliminary calculation of constant auxiliary parameters is made: $H_{C T}=R_{C T} \operatorname{ctg} \alpha_{C}$-the distance from $P_{T}$ to the upper base of the receiving cone; $d_{T X, \text { min }}=H_{C T}+R_{S} \sin \alpha_{C}$ and $d_{T X, \text { max }}=d_{T X, \text { min }}+\left(R_{C B}-R_{C T}\right) \operatorname{ctg} \alpha_{C}-$ minimal and maximal distances from the $P_{S}$ point to the $P_{T}$ point along the cone axis provided its contact with the sphere; $d_{L, \text { min }}=R_{C T}-R_{S} \cos \alpha_{C}$ and $d_{L, \text { max }}=d_{L, \text { min }}+\left(R_{C B}-R_{C T}\right)-$ minimal and maximal distances from the sphere center $P_{S}$ to the cone axis provided its contact with the sphere; $n_{C X}=\sin \alpha_{C}, n_{C L}=\cos \alpha_{C}-$ constant axial and lateral components of the contact reaction unit vector normal to the cone surface.

Geometric contact parameters are calculated as follows.

1. From the sphere center $P_{S}$, the distances are calculated: $d_{L}=\sqrt{y_{S}^{2}+z_{S}^{2}}$ to the axis, and $d_{T X}=x_{T}-x_{S}$ to the top of the receiving cone along its axis. When one of the conditions $d_{L}<d_{L, \text { min }}, d_{L}>d_{L, \max }$, and $d_{T X}<d_{T X, \min }$ or $d_{T X}>d_{T X, \max }$ is met the search of a contact point of this type is terminated. 


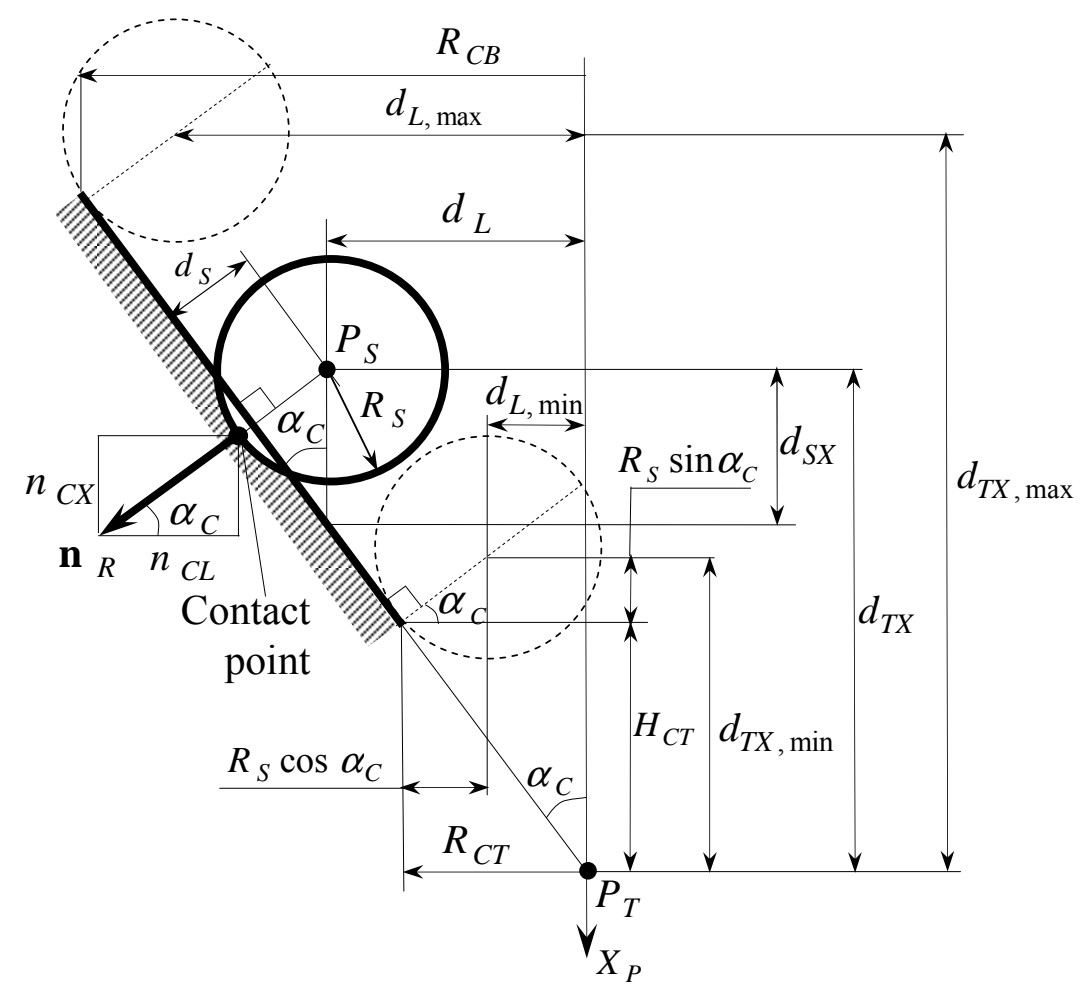

Fig 2. A sphere to a truncated cone surface contact model

2. The distance is calculated from the sphere center $P_{S}$ to the cone surface along its axis $d_{S X}=d_{T X}-d_{L} \operatorname{ctg} \alpha_{C}$ and along the normal to its surface $d_{S}=d_{S X} \sin \alpha_{C}$. If $d_{S}>R_{S}$ then a contact of this type is missing, and determination of its parameters is terminated.

3. The penetration value $\Delta_{n}=R_{S}-d_{S}$ is calculated along the normal to the cone surface. If it exceed the maximal allowable limit, i.e. $\Delta_{n}>\Delta_{n, \max }$, then the simulation is terminated with a corresponding diagnostic message.

4. If $0<\Delta_{n}<\Delta_{n \text {, max }}$ then a contact point between the sphere and the receiving cone is obtained. It is given a current number $k$. The reaction unit vector $\mathbf{n}_{R, k}^{(P)}=\left[n_{C X}, n_{Y}, n_{Z}\right]^{T}$ has one constant component $n_{X}=\sin \alpha_{C}$ and two variable components $n_{Y}=n_{C L}\left(y_{S} / d_{L}\right)$ and $n_{Z}=n_{C L}\left(z_{S} / d_{L}\right)$.

Geometric contact parameters for the pair of geometric primitives sphere - circle, sphere - cylinder, sphere - plane are calculated similarly using preliminarily calculated characteristic distances from the sphere center corresponding to such contact types: to the axis of the circle, cone or cylinder, to the top of the cone or plane. Formulas for determining parameters of contact of individual points representing segment extremities with passive conic and cylindrical surfaces are derived from similar relations for the sphere, provided $R_{S}=0$.

In the algorithms presented below for geometric primitives in a segment form, well-known formulas of analytical geometry are used [5]. In particular, a line passing through points $P_{1}=\left(x_{1}, y_{1}, z_{1}\right)$ and $P_{2}=\left(x_{2}, y_{2}, z_{2}\right)$ is described by the parametrical equation $x=x_{1}+l t$, $y=y_{1}+m t, z=z_{1}+n t$, i.e. the initial point $P_{1}$ and the guide vector $\mathbf{L}=[l, m, n]^{T}$ with components $l=x_{2}-x_{1}, \quad m=y_{2}-y_{1}, \quad n=z_{2}-z_{1}$. If this line crosses a plane 
$A x+B y+C z+D=0$ in a point $P_{C}$ then the corresponding value of the parameter $t_{C}=-\left(A x_{1}+B y_{1}+C z_{1}+D\right) /(A l+B m+C n)$, and the point $P_{C}$ has coordinates $x_{C}=x_{1}+t_{C} l, y_{C}=y_{1}+t_{C} m, z_{C}=z_{1}+t_{C} n$. It belongs to the segment between $P_{1}$ and $P_{2}$, provided $t_{C} \in[0,1]$.

Parameters of the plane passing through a segment $\mathbf{L}_{1}$ parallel to another segment $\mathbf{L}_{2}$ and parameters of the plane passing through the segment $\mathbf{L}_{1}$ perpendicular to the plane $A x+B y+C z+D=0$ are determined from structure-like equations

$$
\left|\begin{array}{ccc}
x-x_{1} & y-y_{1} & z-z_{1} \\
l_{1} & m_{1} & n_{1} \\
l_{2} & m_{2} & n_{2}
\end{array}\right|=0 \text { and }\left|\begin{array}{ccc}
x-x_{1} & y-y_{1} & z-z_{1} \\
l_{1} & m_{1} & n_{1} \\
A & B & C
\end{array}\right|=0
$$

\subsection{Calculation of a line segment to a circle line contact parameters.}

A circle with a radius $R_{C}$ and a center $P_{B}=\left(x_{B}, y_{B}, z_{B}\right)$ is the base of a truncated cone or cylinder. The top of the cone or the center of the other base of the cylinder are determined by the point $P_{T}=\left(x_{T}, y_{T}, z_{T}\right)$. The segment is represented by the vector $\mathbf{L}_{i}=\left[l_{i}, m_{i}, n_{i}\right]^{T}$ with the initial point $P_{1, i}$ (see Fig. 3).

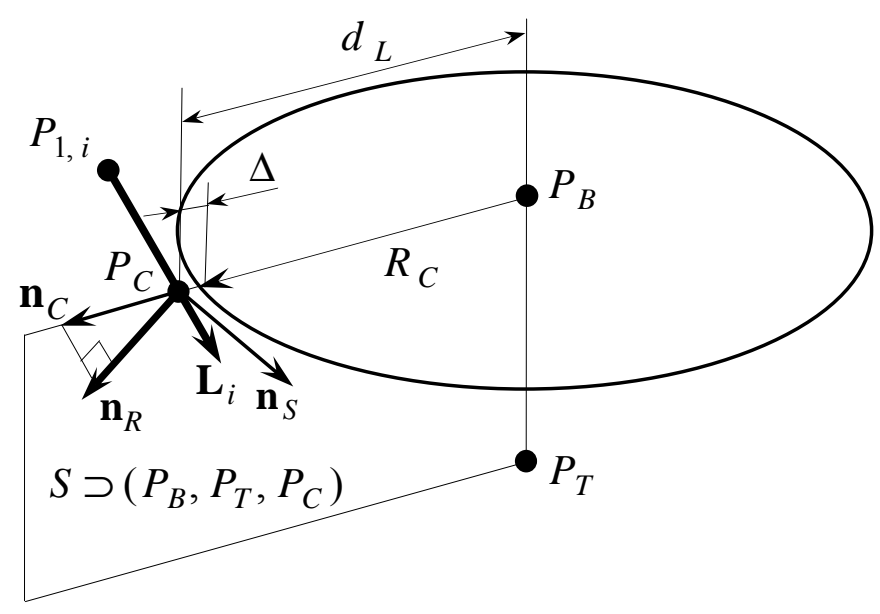

Fig. 3. A line segment and a circle line contact geometrical model

To determine the geometric contact parameters, the following items are calculated sequentially:

1. Parameters $A, B, C, D$ of the circle plane passing through $P_{B}$ perpendicular to the segment $P_{B} P_{T}$ of the cone axis or cylinder: $A=l=x_{T}-x_{B}, \quad B=m=y_{T}-y_{B}$, $C=n=z_{T}-z_{B}, D_{C}=-\left(l x_{B}+m y_{B}+n z_{B}\right)$.

2. The parameter $t_{C}$ corresponding to the cross point $P_{C}$ of the pointed segment $\mathbf{L}_{i}$ and the circle plane; if $t_{\mathrm{C}} \not \subset[0,1]$ then the segment does not cross the circle plane, and determination of contact parameters of this type is terminated; 
3. The coordinates of the point $P_{C}$ and the distance $d_{L}$ from it to the circle center $P_{B}$ (if $d_{L} \leq R_{C}$ then the segment does not cross the circle, and determination of its contact parameters of this type is terminated);

4. The components of the guide vector $\mathbf{n}_{S}=\left[A_{S}, B_{S}, C_{S}\right]^{T}$ of a plane $S$ passing through three points $P_{C}, P_{B}$ and $P_{T}$, i.e. the components of a vector parallel to a tangent to the circle in the point of its crossing the segment $P_{B} P_{C}$

$$
\begin{aligned}
& A_{S}=\left(y_{B}-y_{C}\right)\left(z_{T}-z_{C}\right)-\left(y_{T}-y_{C}\right)\left(z_{B}-z_{C}\right), \\
& B_{S}=-\left[\left(x_{B}-x_{C}\right)\left(z_{T}-z_{C}\right)-\left(x_{T}-x_{C}\right)\left(z_{B}-z_{C}\right)\right], \\
& C_{S}=\left(x_{B}-x_{C}\right)\left(y_{T}-y_{C}\right)-\left(x_{T}-x_{C}\right)\left(y_{B}-y_{C}\right) ;
\end{aligned}
$$

5. The unit vector $\mathbf{n}_{R}$ of the reaction in the contact point as a result of normalization of the components of the vector $\mathbf{L}_{i} \times \mathbf{n}_{S}$ with fulfillment of the condition $\mathbf{n}_{R, x}>0$.

6. The unit vector $\mathbf{n}_{C}=\left[0, y_{C} / d_{L}, z_{C} / d_{L}\right]^{T}$ of the circle radius passing through the point $P_{C}$ allows to determine the contact penetration value $\Delta_{n}=\left(d_{L}-R_{C}\right)\left(\mathbf{n}_{C}^{T} \mathbf{n}_{R}\right)$ along the vector $\mathbf{n}_{R}$.

If $\Delta_{n} \leq \Delta_{n, \max }$ then $P_{C}$ is the contact point, otherwise the simulation is terminated with a corresponding diagnostic message.

\subsection{Calculation of contact parameters for lateral borders of guide petals}

Inclined lateral borders of guide petals are represented by pointed segments with initial points located on the lower bases of the petals. If the limitations on the initial contact conditions are met the extremity of the pointed segment $\mathbf{L}_{\mathrm{A}}$ of the active guide petal border is always outside the nearest passive petal, and does not go beyond the limits of the nearest lateral border of this petal (see Fig. 4.a).

This allows to implement the following algorithm of determining geometric parameters of contact of pointed segments $\mathbf{L}_{\mathrm{A}}$ and $\mathbf{L}_{\mathrm{P}}$ of the borders of the active and passive guide petals. First (see Fig. 4.b), sequentially, as follows below, goes the calculation of the parameters of

1. the plane $\mathbf{S}_{\mathrm{PP}}$ passing through $\mathbf{L}_{\mathrm{A}}$ parallel to $\mathbf{L}_{\mathrm{P}}$;

2. the plane $\mathbf{S}_{\mathrm{PA}}$ passing through $\mathbf{L}_{\mathrm{P}}$ parallel to $\mathbf{L}_{\mathrm{A}}$;

3. the plane $\mathbf{S}_{\mathrm{NP}}$ passing through $\mathbf{L}_{\mathrm{A}}$ normal to $\mathbf{S}_{\mathrm{PP}}$;

4. the plane $\mathbf{S}_{\mathrm{NA}}$ passing through $\mathbf{L}_{\mathrm{P}}$ normal to $\mathbf{S}_{\mathrm{PA}}$.

5. the parameter $t_{\mathrm{CA}}$ that corresponds, for the segment $\mathbf{L}_{\mathrm{A}}$, to the point $\mathrm{C}_{\mathrm{A}}$ of its intersection with the plane $\mathbf{S}_{\mathrm{NA}}$

6. the parameter $t_{\mathrm{CP}}$ that corresponds for the segment $\mathbf{L}_{\mathrm{P}}$ to the point $\mathrm{C}_{\mathrm{P}}$ of its intersection with the plane $\mathbf{S}_{\mathrm{NP}}$.

If $t_{\mathrm{CA}} \not \subset[0,1]$ or $t_{\mathrm{CB}} \not \subset[0,1]$ the segments do not cross, and the calculation of contact parameters is terminated. Otherwise, the segment $C_{A} C_{P}$ crosses $\mathbf{L}_{A}$ and $\mathbf{L}_{P}$ and is normal to them. Its length is equal to the minimal distance between these segments. 


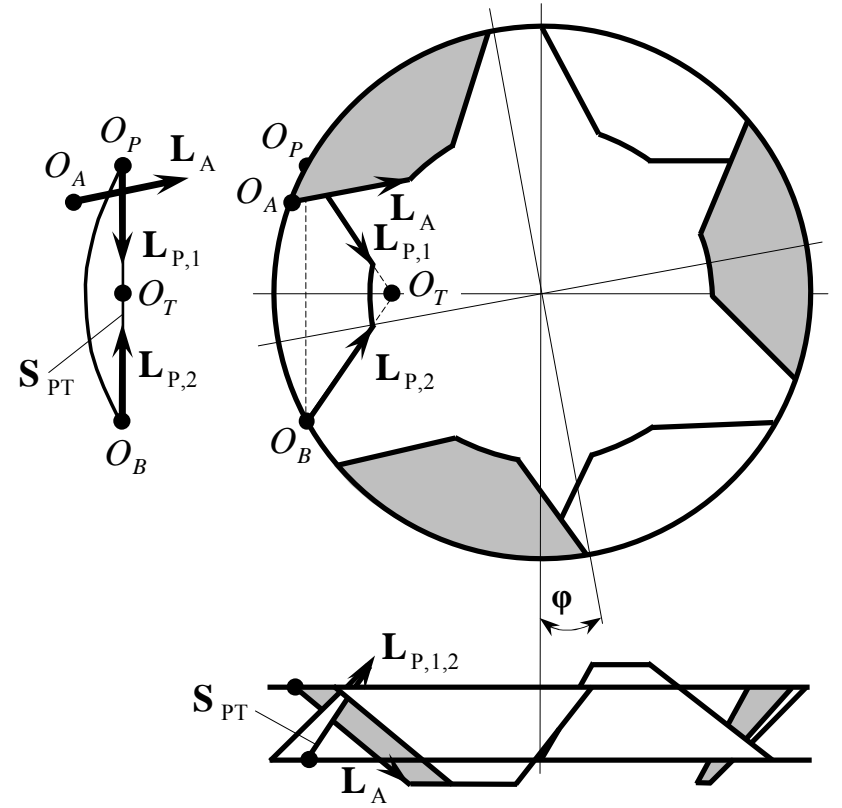

a

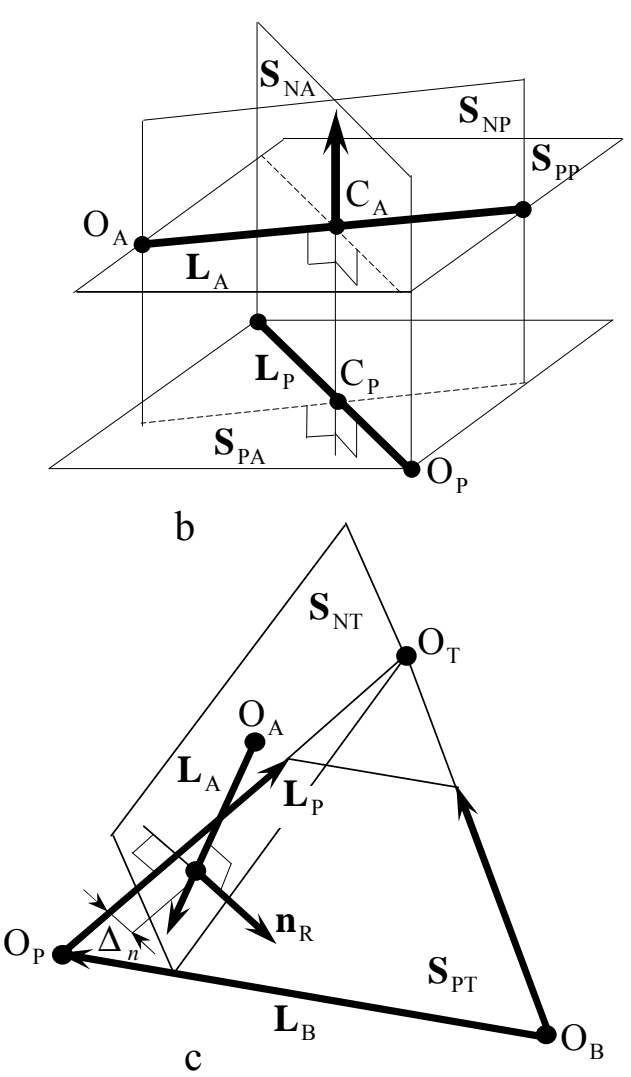

Fig. 4. Contact of lateral faces of guide petals $\mathrm{a}$ - position of contacting faces relative to the guide petals surface with an axial turn of the ring; $\mathrm{b}$ - building of the common normal to the line segments; $\mathrm{c}-$ determination of penetration at the border contact

To determine the fact of contact penetration, an additional geometric element is used: an isosceles triangle formed by a continuation of the pointed segments of both borders of the passive guide petal until their intersection on the top $\mathrm{O}_{\mathrm{T}}$. Its base that joins the initial points of the border segments is specified by the pointed segment $\mathbf{L}_{\mathrm{B}}$ with the initial point $\mathrm{O}_{\mathrm{B}}$ (see Fig. 4c). The coordinates of the points $\mathrm{O}_{\mathrm{T}}$ and $\mathrm{O}_{\mathrm{B}}$, the parameters of the plane $\mathbf{S}_{\mathrm{T}}$ of the characteristic triangle and the segment $\mathbf{L}_{\mathrm{B}}$, as well as the parameters of the border segments of the passive guide petal, are constant values in the coordinate system $X_{P} Y_{P} Z_{P}$.

To determine the fact of penetration, i.e. contact, sequentially, as follows below, goes the calculation of the parameters of

1. the line segment $\mathbf{L}_{\mathrm{TA}}$ passing through the points $\mathrm{O}_{\mathrm{T}}$ and $\mathrm{C}_{\mathrm{A}}$;

2. the plane $\mathbf{S}_{\mathrm{NT}}$ passing through $\mathbf{L}_{\mathrm{TA}}$ normal to $\mathbf{S}_{\mathrm{T}}$;

3. $t_{\mathrm{CB}}$ that corresponds to the intersection point of the segment $\mathbf{L}_{\mathrm{B}}$ with the plane $\mathbf{S}_{\mathrm{NT}}$.

If $t_{\mathrm{CB}} \not \subset[0,1]$ then no contact is available. Otherwise, a contact penetration $\Delta_{n}$ occurs, its value is equal to the length of the segment $\mathrm{C}_{\mathrm{A}} \mathrm{C}_{\mathrm{P}}$. If it exceeds the allowable limit $\Delta_{n \text {, max }}$ then the simulation process is terminated with a corresponding diagnostic message. Otherwise, the point $\mathrm{C}_{\mathrm{A}}$ is considered a contact point, and the parameters of the normal 
reaction line of action are calculated as $\mathbf{L}_{\mathrm{N}}=\mathbf{L}_{\mathrm{A}} \times \mathbf{L}_{\mathrm{P}}$. The contact reaction unit vector is determined as a result of normalization of the vector $\mathbf{L}_{\mathrm{N}}$, provided $\mathbf{L}_{\mathrm{N}, \mathrm{x}}>0$.

\subsection{Calculation of contact interaction forces of guide surfaces.}

In such a case contact interaction forces are determined by the value and velocity of penetration along the reaction unit vector, as well as the velocity of relative motion in the plane tangent to the contacting elements. All parameters of the contact points and contact interaction force are calculated in the coordinate system $X_{P} Y_{P} Z_{P}$ of the passive contacting surface. Primitives of the active surface elements may belong to one or several different links of the docking mechanism. In this case parameters of each primitive are expressed in one of moving coordinates $X_{A, k} Y_{A, k} Z_{A, k}$ and converted into $X_{P} Y_{P} Z_{P}$ with the direction cosine matrix $\mathbf{A}_{A P, k}$. The velocities of the passive and the active geometric primitives in a $i-t h$ contact point are equal

$$
\begin{aligned}
& \mathbf{v}_{P C, i}^{(P)}=\mathbf{v}_{P}^{(P)}+\boldsymbol{\omega}_{P}^{(P)} \times \mathbf{r}_{P C, i}^{(P)}, \\
& \mathbf{v}_{A C, i}^{(P)}=\mathbf{A}_{A P, k}\left(\mathbf{v}_{A}^{(A, k)}+\left(\boldsymbol{\omega}_{A}^{(A, k)}+\boldsymbol{\omega}_{A, i}^{r e l(A, k)}\right) \times \mathbf{r}_{A C, i}^{(A, k)}+\mathbf{v}_{A C, i}^{r e l(A, k)},\right.
\end{aligned}
$$

where $\mathbf{v}_{P}^{(P)}, \boldsymbol{\omega}_{P}^{(P)}$ и $\mathbf{v}_{A}^{(A, k)}, \boldsymbol{\omega}_{A}^{(A, k)}-$ absolute velocities $X_{P} Y_{P} Z_{P}$ and $X_{A, k} Y_{A, k} Z_{A, k} ; \mathbf{r}_{P C, i}^{(P)}$ and $\mathbf{r}_{A C, i}^{(A, k)}$ - radius vectors of the contact point relative to $X_{P} Y_{P} Z_{P}$ and $X_{A, k} Y_{A, k} Z_{A, k}$, $\mathbf{v}_{A C, i}^{r e l(A, k)}, \boldsymbol{\omega}_{A, i}^{r e l(A, k)}$ - velocities of the active geometric primitive relative to $X_{A, k} Y_{A, k} Z_{A, k}$.

Velocity $\dot{\Delta}_{n, i}$ of penetration along the contact reaction vector $\mathbf{n}_{R}^{(P)}$, tangential velocity $\mathbf{v}_{T, i}^{(P)}$ in the contact point and its unit vector $\mathbf{n}_{T, i}^{(P)}$ are equal to

$$
\begin{aligned}
& \dot{\Delta}_{n, i}=\left(\mathbf{v}_{A C, i}^{(P)}-\mathbf{v}_{P C, i}^{(P)}\right)^{T} \mathbf{n}_{R}^{(P)}, \\
& \mathbf{v}_{T, i}^{(P)}=\mathbf{v}_{A C, i}^{(P)}-\mathbf{v}_{P C, i}^{(P)}-\dot{\Delta}_{n, i} \mathbf{n}_{R}^{(P)}, \quad \mathbf{n}_{T, i}^{(P)}=\mathbf{v}_{T, i}^{(P)} /\left|\mathbf{v}_{T, i}^{(P)}\right| .
\end{aligned}
$$

Respectively, forces $\mathbf{f}_{P C, i}^{(P)}$ and $\mathbf{f}_{A C, i}^{(P)}$ applied on the passive and active mechanisms in a $i$-th contact point are determined by the relations

$$
\begin{aligned}
& \mathbf{f}_{P C, i}^{(P)}=\left(k_{S, i} \Delta_{n, i}+c_{D, i} \dot{\Delta}_{n, i}\right)\left(\mathbf{n}_{R}^{(P)}-k_{F r, i} \mathbf{n}_{T, i}^{(P)}\right), \\
& \mathbf{f}_{A C, i}^{(P)}=\left(k_{S, i} \Delta_{n, i}+c_{D, i} \dot{\Delta}_{n, i}\right)\left(-\mathbf{n}_{R}^{(P)}-k_{F r, i} \mathbf{n}_{T, i}^{(P)}\right),
\end{aligned}
$$

where $k_{S, i}, c_{D, i}-$ contact stiffness and damping factors, $k_{F r, i}-$ friction factor in the contact point.

Moments of these forces relative to $X_{P} Y_{P} Z_{P}$ and $X_{A, k} Y_{A, k} Z_{A, k}$ are modulo different even without the friction force due to the difference of the vectors $\mathbf{r}_{P C, i}^{(P)}$ and $\mathbf{r}_{A C, i}^{(A, k)}$.

\section{REDUSING OF REDUNDAND CALCULATIONS, USAGE OF DICHOTOMY METHOD}

Above, on describing the algorithms for different pairs of geometric primitives, the simplest method of increasing calculation efficiency was demonstrated, i.e. termination of calculations if required contact conditions are not met. 
A more complex approach is based on a substantial analysis and elimination of incompatible types of contact. In particular, for a sphere to a receiving cone contact, it is not required to analyze interaction of the sphere with the inlet border (circle) and the receiving cone cylinder. It is not required to consider the second border of the guide petal if there is a contact with one border. Contact of the cone surface of one of the guide petals and the sphere of the counter receiving cone base excludes a similar type interaction for other guide petals of the same contact surface.

To determine contact points and parameters of surfaces represented by a finite set of geometric primitives, a direct search over the primitives can be avoided. For this, during initialization of the mathematical model, a description $2^{n}+1$ of approximating geometric primitives - generatrix segments for a cone surface and spheres for a torus - is automatically generated in each of several sectors of these surfaces. The number $n$ depends on the given accuracy of solution and determines the number of steps needed for a quick isolation of the approximating primitive, most distant from the axis of the receiving cone, circle or cylinder, using a dichotomy method [6] implemented in the following way.

At a successive iteration step, characteristic distances from the center of the counter line or axis of the second-order surface are determined for two extreme and one middle geometric primitives of the current sector with indexes $i_{B}, i_{E}, i_{M}$ respectively. For generatrix segments of a cone surface these are distances between points of their intersection with the circle plane and the center of this circle; for spheres approximating a torus these are distances between the surfaces of the approximating sphere and the counter geometric primitive, i.e. a circle line, the surface of a cone or cylinder.

At the first iteration, the index values of the extreme and middle primitives are equal to $i_{B}=1, i_{E}=i_{B}+2^{n}, i_{m}=i_{B}+2^{n-1}$. At the last iteration, among the three primitives with the indexes $i_{B}, i_{M}=i_{B}+2^{0}=i_{B}+1$, and $i_{E}=i_{B}+2^{1}=i_{B}+2$ one is selected with the maximal characteristic distance, contact parameters are determined for it, and the iteration process is terminated. The total number of its steps does not exceed $n$. Uniqueness of solution is secured by the convex form of as minimum one of the contacting elements and the linear form of the other.

If characteristic distances for all the three primitives are exactly equal it means that the axis of the cone surface or the torus coincides with the axis of the counter primitive. In this case the contact parameter is determined for the middle primitive. If this condition is not fulfilled two out of the three primitives are selected with maximal characteristic distances, the indexes $i_{B}$ and $i_{E}$ are assigned new values, and the search area is narrowed twice. An index $i_{M}=\left(i_{B}+i_{E}\right) / 2$ is calculated for a new middle primitive, and the iteration process goes to a next step.

When determining contact parameters for any primitives with the maximal characteristic distance, penetration and contact may not be available. If a geometric primitive with the maximal characteristic distance is isolated at a boundary of a sector then a possibility must be analyzed for contact in the adjacent sector as well. If this primitive is interior then the rest of the sectors may not be considered.

Only those approximating geometric primitives of the surface element of the active assembly that are isolated with the dichotomy method are converted into the coordinate system $X_{P} Y_{P} Z_{P}$.

Fig. 5 shows a simplified example of only three iteration steps of the determination of the most circle center remote point of intersection of the circle plane and the generatrices of a truncated cone. At the first step the cone surface sector generatrices have indexes 
$i_{B}(1), i_{E}(1), i_{M}(1)$. The characteristic distance for a $k$-th iteration is equal to $\max \left\{d_{B}(k), d_{M}(k), d_{E}(k)\right\}$, where $d_{B}(k), d_{M}(k), d_{E}(k)-$ are distances between the center of a circle with the radius $R$ and intersection points of the circle plane with segments of the truncated cone generatrices with indexes $i_{B}(k), i_{E}(k), i_{M}(k)$. The characteristic distance of the most center remote point of intersection is equal $d_{M}(3)<R$, which indicates absence of penetration and contact point.

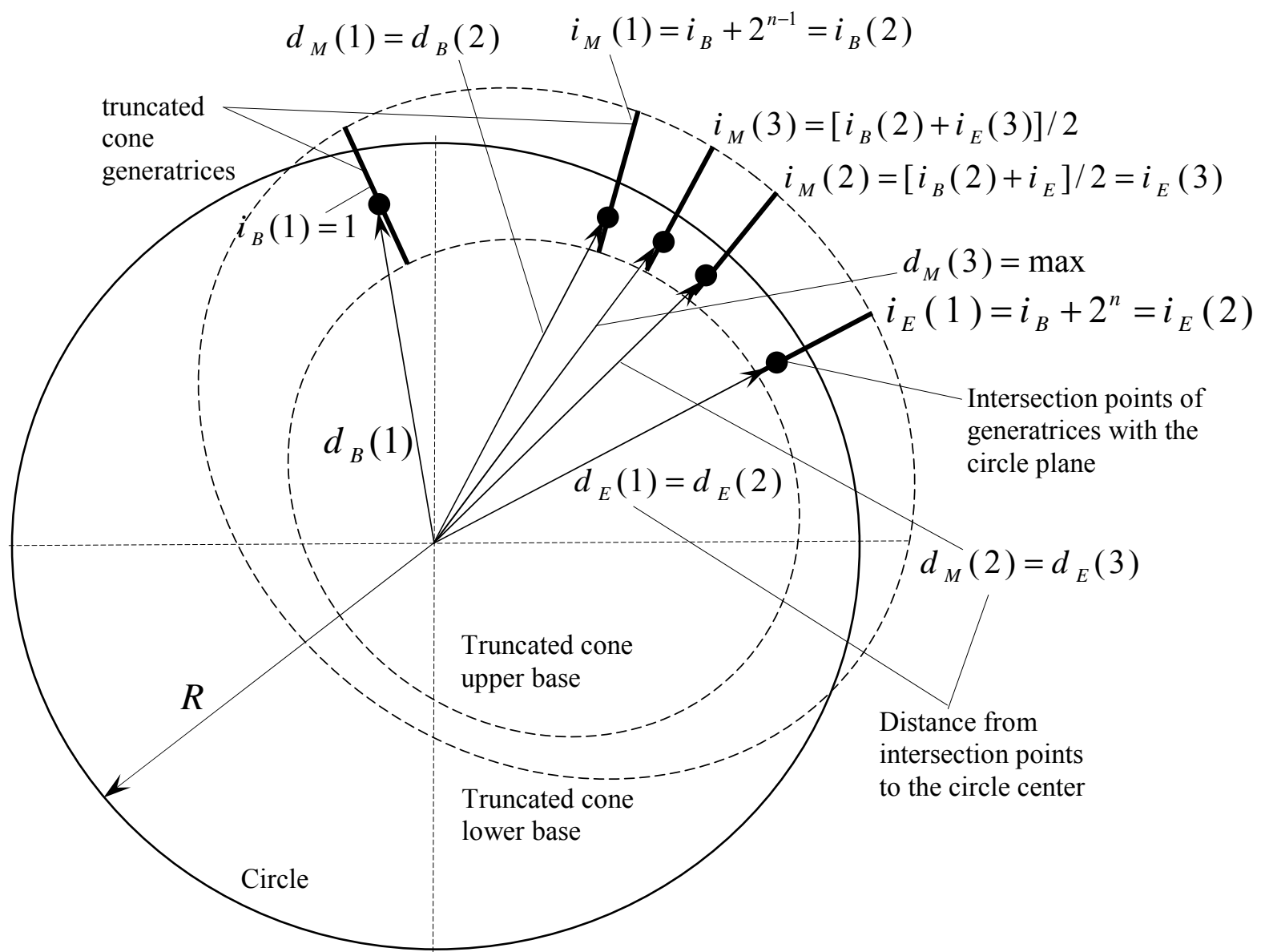

Fig. 5. Iterative determination of the most circle center remote point of intersection of the circle plane and the generatrix of the truncated cone (three iteration steps are shown).

Guide petals represent cone surface fragments, whose length of generatrix segments steadily changes in the areas of inclined lateral borders. Due to such a form of petals and presence of voids in the cone surface, it might exceed the bounds of the counter geometric primitive (circle). With that, generatrix lines in the areas of lateral faces may cross the circle, but their segments of a limited length will not contact the circle plane. Parameters of such a complex contact interaction are determined using the following modification of the above described algorithm for a next in turn guide petal.

At the first step, the dichotomy method is used to determine a generatrix segment with the index $i_{\text {Max }}$ that has the maximal value of the characteristic distance $\Delta$. If $\Delta<=0$ then contact of the guide petal is impossible, and determination of its parameters is terminated. Otherwise, if for a segment with the index $i_{\text {Max }}$ the parameter of intersection with the circle 
plane meets the condition $t_{C}\left(i_{\text {Max }}\right) \in[0,1]$ then contact parameters are calculated according the above described algorithm.

If $t_{C}\left(i_{M a x}\right)>1$ or $t_{C}\left(i_{M a x}\right)<0$ then the generatrix and the circle plane intersection point, which is most remote from the circle center, does not belong to the approximating segment of the guide petal. However in this case contact of adjacent approximating segments is possible.

For an identification of their contact, a sequential search is done in the direction of its possibility increase. The length of guide petal generatrix segments can change nly steadily; therefore, starting from a segment with index $i_{\text {Max }}$, sequentially selected are those whose $t_{C}$ value decreases if $t_{C}\left(i_{\text {Max }}\right)>1$, and it increases if $t_{C}\left(i_{\text {Max }}\right)<0$. Contact is not available if

- for a next in turn segment the condition $t_{C} \in[0,1]$ is met, but the characteristic distance $\Delta<=0$ for its point of intersection with the circle plane;

- the extreme (first or last) approximating segment of the guide petal has been searched but no penetration into the circle identified.

If for a next in turn approximating segment of the generatrix the condition $t_{C} \in[0,1]$ is met and penetration into the circle occurs then its contact parameters are determined according to the above described relations for a line segment and a circle line, and the analysis of the next guide petal is terminated.

This algorithm requires a significantly smaller scope of calculations than a direct search over all approximating segments of a guide petal cone surface.

\section{METHOD OF REACTION FORCE DETERMINATION FOR SECOND CLASS CONTACT MODELS ILLUSTRATED ON MECHANICAL LATCHES}

The contact reaction force in this case is determined by deformation of the spring acting on the contacting element that has one degree of freedom relative to the buffer link of the docking mechanism or docking assembly body. The notion of penetration is not used, which allows a precise fulfillment of geometric contact conditions.

The position of a spring-loaded element with one degree of freedom of a relative motion at contact is determined iteratively with the dichotomy method. But in this case a new search sector, after dichotomization of the previous one, is selected on the condition of a sign difference of the characteristic distances between contacting elements at its bounds.

The initial bounds of the solution search interval correspond to the element positions at the initial and the most deformed spring state. At the model initialization step, this interval is divided into $2^{n}$ equal parts corresponding to $2^{n}+1$ intermediate positions. The number $n$ is determined by a given solution accuracy that is limited theoretically only by real number representation accuracy in the computer. For a practically needed solution accuracy, the number of iterations is relatively small due to power dependence. For each possible position, the spring resistance force or moment, contacting element geometric parameters, and parameters of the reaction unit vector depending on the type of contact are calculated. That slightly increases the required memory size, but significantly increases the computation speed because during the iteration process pre-calculated parameters corresponding to possible positions of the element are just converted to the common calculation coordinate system. Therewith, only parameters for the positions selected during the dichotomy process are subject to conversion. Uniqueness of solution, as before, is secured by the form of contacting elements.

During simulation, for each spring-loaded element in the contacting pair within a given interval of its relative motion, characteristic distances to the counter element are calculated. If the characteristic distance for the unstrained spring position is negative then contact between 
the elements does not occur. If at the extremities of the initial interval the characteristic distances have unlike signs then a position is searched, at which the characteristic distance is positive and within the required accuracy.

Algorithms of determination of distances between contacting elements are similar to the above described for guide surface simulation. For a calculated with a given accuracy relative latch position, the resistance force is already determined at the model initialization stage. For the pair of geometric primitives 'line segment - circle', pre-calculation is made for components of the reaction unit vector normal to the first characteristic segment of the latch. For the pair of geometric primitives 'point - conic surface', components of the reaction unit vector are determined in the same way as for contact between a sphere and a conic surface. It should be noted that, as for the calculation of contact parameters of guide surfaces, the algorithms are developed provided the presence of only those linear and angular docking mechanism misalignments that meet given limitations.

Contacting spring-loaded elements with one degree of freedom of relative motion are latches, contact sensors, passive hooks of the interface pressurization mechanism locks.

The geometry of a mechanical latch can be roughly described by two segments 1 and 2 corresponding to its direct and inverse surfaces. Segment 1 (direct surface) is contacting with counter elements before a latch operation, and segment 2 (inverse surface) is contacting with other counter elements after its operation. Latch operation conditions are expressed by inequalities that must be satisfied with the coordinates of the segment 1 extremity.

For a rotary-type latch (see Fig. 6), before its operation, the pairs of contacting geometric primitive are: point (segment extremity) - conic surface, line segment - circle (receiving socket inlet border). To describe a translation-type latch, only one pair of primitives point (segment extremity) - conic surface is sufficient.

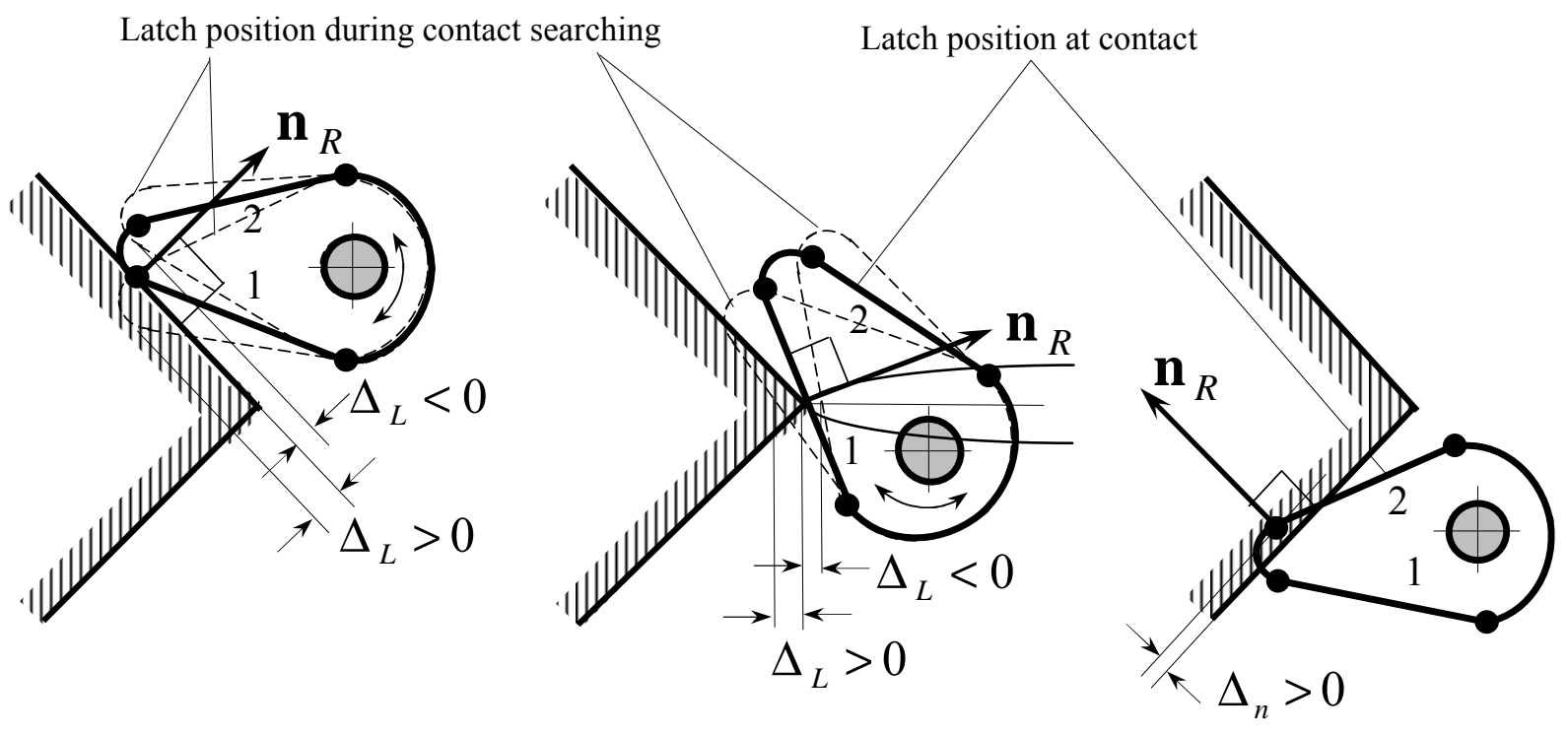

Fig. 6. Characteristic distances 1 and 2 for the description of rotary-type latch contacts:

$\Delta_{L}-$ characteristic distances calculated until latch operation when determining its contact with a cone or circle

$\Delta_{n}-$ penetration determined after its operation at contact with a mechanical stop

After latch operation (engagement), its direct rotation is impossible due to the absence of external forces applied to the direct surface (segment 1). Rotation in the inverse direction at a 
contact of the inverse surface (segment 2) with a stop of the counter assembly is impossible due to the presence of the own mechanical stop of the latch. Due to the constant relative position of the latch, its contact parameters can be determined only using the penetration notion. In this case the pairs of contacting geometric primitives are: point (segment extremity) - conic surface (of the counter element stop) and line segment - circle (border of the counter element stop). Algorithms for calculation of contact parameters for these cases are already described above.

Sensors contacting with guide surfaces and docking assembly bodies are also springloaded elements with one degree of freedom of relative motion. As well as latches, they can be rotary-type or translation-type. Their contacting surfaces can be represented accurately enough by a sphere. Their contact parameters are determined in the same way as for latches before engagement.

Similarly, the dichotomy iteration method can be used to determine contact parameters and reaction forces for interaction of each pair of hooks, i.e. the active controllable hook and the passive spring-loaded hook that has one degree of freedom. During rigid joining of docking assemblies, movements of these links have a lower amplitude, and the contacting surfaces have a more complex form that must be described much more accurately to assess engagement sensitivity to tight hook size tolerances. Models of such a contact interaction type are currently under development.

\section{VERIFICATION OF CONTACT INTERACTION MATH MODEL}

Verification includes a testing of algorithms and software operation, as well as a validation of geometric and dynamic parameters of the model.

Stand-alone testing of contact interaction simulation algorithms and software is performed before their integration into the general docking sequence model. However at this phase, it is very difficult to discover all behavioral features of the chosen analytical model. Therefore, the testing capability is also available during the dynamic process simulation. As the software code realizes a very complex model, utilization of a universal debugger is minimized. The simulation software has a capability of recording all model parameters into a file in a convenient format. To make the file size appropriate for analysis, the testing mode can be activated for any shortest possible time space, up to an individual integration step. Within this time space, individual indicators can be installed to initiate testing of a particular algorithm. Simulation can be abnormally terminated if the algorithm itself reveals a violation of contact condition limitations, allowable limit of the penetration value, or in case of a system error. Based on the simulation time of such occurrence and the error type, which are recorded in the simulation log, the testing time frame and indicators are selected for a repeated simulation and analysis. This method more than once gave information for a size correction of individual contacting elements even after a long-term use of the simulation software.

During the docking sequence, until a rigid connection of the assemblies, most contacts are damped by the docking mechanism that is more compliant than structural elements of the assemblies or the spacecraft. Therefore, it is sufficient to apply the design values of contact stiffness. For particular low-damping contacts, specific static and dynamic tests are done. A final model verification is done based on the results of a quasi-flight testing on a hybrid 6DOF dynamic test facility with real docking assemblies and an approaching spacecraft motion math model. These test results are saved as time function measured forces and moments of contact interaction, spacecraft relative motion parameters, readings of mechanism sensors. During a real docking, sensor readings and spacecraft angular velocities are measured and recorded. The model performance criterion is a compliance of all this data with the design values. 


\section{CONCLUSION}

Development of the above described algorithms started more than twenty years ago following the implementation of the new, at that time, approach to the description of docking mechanisms by rigid-body system dynamic equations. They ensure a real-time simulation of contact interaction for different types of docking interfaces: probe-cone, androgynous and specialized berthing units. Adequacy of these models used as part of integrated models of docking assemblies and sequences was repeatedly proved by the compliance of simulation results with ground and flight test data.

\section{REFERENCES}

[1] Course of theoretical mechanics: Course book for universities / V.I. Drong, V.V. Dubimnin, M.M. Ilyin et al.; Edited by K.S. Kolesnikov. 3-d edition, M.: MBSTU press, 2005.

[2] J.W. Baumgarte,. Stabilization of constraints and integrals of motion in dynamical systems.- Computer Methods in Applied Mechanics and Engineering, Vol.1, 1-16, 1972.

[3] G.W. Gear, Differential-algebraic Equations. - NATO ASI Series, Vol. 9. Computer Aided Analysis and Optimization of Mechanical System Dynamics./ Edited by E.J. Haug, Springer-Verlag, Berlin-Heidelberg, 1984.

[4] L.R. Petzold, Computational challenges in mechanical system simulation. - ComputerAided Analysis of rigid and flexible Mechanical Systems, Kluwer Academic Publishers, Netherlands, 483-499, 1994.

[5] M.Y. Vygodskiy, Higher mathematics handbook. - M.: Technical-theoretical literature main press, 1956.

[6] B.P. Demidovich, I.A. Maron, Fundamental computational mathematics. M.: Physmathgiz, 1963. 